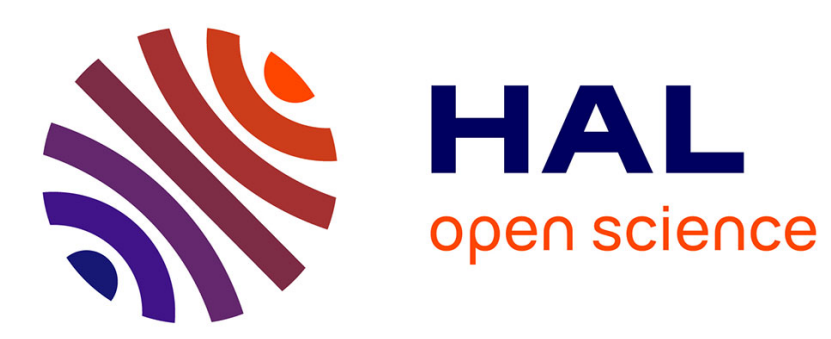

\title{
Conception théorique d'un nouveau modulateur électrooptique sur GaAs/AlGaAs à largeur de bande commandée en tension
}

D. Rauly, E. Pic, S. Tedjini

\section{- To cite this version:}

D. Rauly, E. Pic, S. Tedjini. Conception théorique d'un nouveau modulateur électrooptique sur GaAs/AlGaAs à largeur de bande commandée en tension. Revue de Physique Appliquée, 1987, 22 (11), pp.1585-1594. 10.1051/rphysap:0198700220110158500 . jpa-00245711

HAL Id: jpa-00245711

https://hal.science/jpa-00245711

Submitted on 1 Jan 1987

HAL is a multi-disciplinary open access archive for the deposit and dissemination of scientific research documents, whether they are published or not. The documents may come from teaching and research institutions in France or abroad, or from public or private research centers.
L'archive ouverte pluridisciplinaire HAL, est destinée au dépôt et à la diffusion de documents scientifiques de niveau recherche, publiés ou non, émanant des établissements d'enseignement et de recherche français ou étrangers, des laboratoires publics ou privés. 
Classification

Physics Abstracts

$42.82-42.60-42.80 \mathrm{E}$

\title{
Conception théorique d'un nouveau modulateur électrooptique sur GaAs/AlGaAs à largeur de bande commandée en tension
}

\author{
D. Rauly, E. Pic et S. Tedjini \\ LEMO UA CNRS 833, 23 rue des Martyrs, 38031 Grenoble, France
}

(Reçu le 11 février 1987, révisé le 12 juin 1987, accepté le 18 juin 1987)

\begin{abstract}
Résumé. - Dans cet article, nous présentons un nouveau type de modulateur électrooptique à onde progressive. L'originalité de ce nouveau composant réside dans l'adjonction d'une tension de commande extérieure qui a pour rôle d'accorder l'indice microonde à l'indice optique : le résultat est un élargissement considérable de la largeur de bande de modulation.
\end{abstract}

\begin{abstract}
This paper addresses a new type of travelling wave electrooptic modulators. The proposed modulator is tuned by an external variable voltage. By this method one may match the microwave index to the optical one. The consequence is a significant enhancement of the bandwidth modulation.
\end{abstract}

\section{Introduction.}

Les télécommunications dans le domaine optique connaissent depuis plusieurs années un véritable essor grâce à l'amélioration des fibres optiques et des sources lasers. Les porteuses optiques peuvent aujourd'hui présenter des raies spectrales très pures [1], ce qui permet de transporter plusieurs porteuses à des longueurs d'onde différentes sur la même fibre, et d'accroître ainsi le débit de l'information.

Une autre façon classique d'améliorer ce débit est d'exploiter au maximum l'énorme largeur de bande potentielle fournie par une porteuse optique. Les dispositifs de modulation les plus prometteurs sont les modulateurs électrooptiques à onde progressive, grâce à l'interaction cumulative entre l'onde optique et l'onde hyperfréquence modulante. Ces modulateurs font donc l'objet, ces dernières années, de nombreuses études focalisées sur l'accroissement de la largeur de bande de modulation [2,3]. Sa limitation a pour origine, entre autres effets (désadaptation d'impédance, etc.) la désadaptation des vitesses de phase des ondes optiques et hyperfréquence.

Dans cet article, nous examinons la faisabilité d'un nouveau modulateur électrooptique, dans lequel la vitesse de phase microonde peut être accordée électroniquement à la vitesse de phase de l'onde optique. Cette particularité importante est dégagée d'une étude théorique rigoureuse des caractéristiques de dispersion et de pertes de ce modulateur dans le domaine microonde.
Cette nouvelle structure réalise la synthèse de deux composants existant déjà et appartenant l'un à la classe des circuits intégrés microonde, l'autre à celle des dispositifs optoélectroniques en optique intégrée.

Le premier est un déphaseur microonde commandé en tension [4]. Son principe de fonctionnement repose sur les propriétés des lignes de transmission déposées sur substrat MIS (Métal-Isolant-Semiconducteur). La vitesse de phase d'un signal microonde se propageant dans une ligne de ce type est très sensible à l'épaisseur de la couche isolante [5]. Plus précisément, la diminution de l'épaisseur de l'isolant relativement à l'épaisseur totale de la structure, entraîne une diminution de la vitesse de phase. Un des effets intervenant dans ce processus est l'augmentation de la capacité de ligne liée à une polarisation interfaciale entre la couche isolante et le semiconducteur. Dans certaines conditions (résistivité du semiconducteur, géométrie de la ligne, mode de propagation), ce phénomène se manifeste depuis les basses fréquences jusqu'à environ $100 \mathrm{GHz}$.

Dans le déphaseur commandé en tension proposé par Neidert et al. [4], la structure MIS est remplacée par un substrat semiconducteur, l'arséniure de gallium (GaAs), sur lequel on a pratiqué un contact Schottky, polarisé en inverse. La zone de déplétion sous la métallisation remplace la couche isolante des structures MIS, et on obtient donc les mêmes propriétés; à la différence près que la vitesse de 
phase de l'onde hyperfréquence peut ici être commandée par la tension de polarisation continue. Ce procédé est bien connu et maîtrisé en microonde. Il a également été décrit avec une technologie silicium [6].

Le deuxième composant est tout à fait classique, lui aussi. C'est un modulateur électrooptique du type interféromètre de Mach-Zehndeer (MZ) à jonction $\mathrm{Y}$, réalisé également en technologie GaAs $[3,7]$. Sa composition est celle d'une hétérostructure GaAs/AlGaAs qui permet de contrôler efficacement le guidage de la lumière. Elle offre suffisamment de souplesse pour s'adapter aux impératifs microondes tout en préservant les propriétés optiques requises.

L'arséniure de gallium, qui est le matériau constituant des deux composants décrits ci-dessus, est finalement bien adapté pour la modulation de lumière large bande, car son indice en microonde $\sqrt{\varepsilon_{\mathrm{r}}}=3,62$ est proche de son indice lumineux $n_{0}=3,59$ à la longueur d'onde optique $\lambda=0,9 \mu \mathrm{m}$ que nous avons choisie. Le réglage de l'indice effectif microonde $n_{\text {meff }}$ à la valeur de l'indice effectif optique $n_{\mathrm{o} \text { eff }}$ est donc possible, à condition de trouver une structure d'électrode appropriée. Cette géométrie, imposée par la configuration $\mathrm{MZ}$, est celle d'une ligne à deux rubans couplés. La diminution simultanée de l'écartement entre les rubans et de leur largeur a pour effet d'abaisser $n_{\mathrm{m} \text { eff }}[8,9]$. Le but est d'optimiser ces dimensions pour que la variation de la tension de polarisation, entre $\mathrm{OV}$ et une valeur négative modérée, entraîne une excursion de $n_{\mathrm{m} \text { eff }}$ de part et d'autre de la valeur de $n_{\mathrm{o} \text { eff }}$. En outre, le confinement de la lumière dans chaque bras de l'interféromètre doit être suffisant afin d'éviter un couplage optique, et ceci avec la structure d'électrode optimisée en hyperfréquence.

En résumé, le modulateur électrooptique proposé doit assurer un fort couplage microonde entre les deux rubans, mais aussi un bon découplage optique entre les deux guides sous ces rubans. Une telle optimisation sous-entend une étude de propagation rigoureuse, aussi bien du point de vue optique que microonde, dans le modulateur.

Le calcul de l'indice effectif lumineux dans les guides optiques est relativement aisé dans la majorité des cas, où des méthodes approchées peuvent s'appliquer [10]. La plupart des structures se ramènent en effet à des géométries plus simples, à cause du confinement de l'énergie lumineuse.

En revanche, l'onde hyperfréquence est beaucoup moins confinée, ce qui oblige à tenir compte de toute la complexité de la structure. Il n'est pas possible ici d'utiliser les formules approchées basées sur l'approximation quasi-TEM, comme pour les modulateurs à structure simple utilisant le niobate de lithium $\left(\mathrm{LiNbO}_{3}\right)$ [11]. Nous avons donc mené l'étude de propagation à l'aide d'une méthode rigoureuse, en l'occurrence la méthode spectrale généralisée (MSG) [12]. La MSG comprend, dans son développement, un traitement analytique préalable qui autorise une grande souplesse pour les géométries des structures à étudier. Elle est donc bien adaptée à notre cas (structure à quatre couches et à deux rubans).

\section{Présentation générale du dispositif.}

La structure de base proposée est illustrée à la figure 1 , où seule la région active de l'interféromètre $\mathrm{MZ}$ a été représentée. Ce dispositif est prévu pour une longueur d'onde optique $\lambda=0,9 \mu \mathrm{m}$.

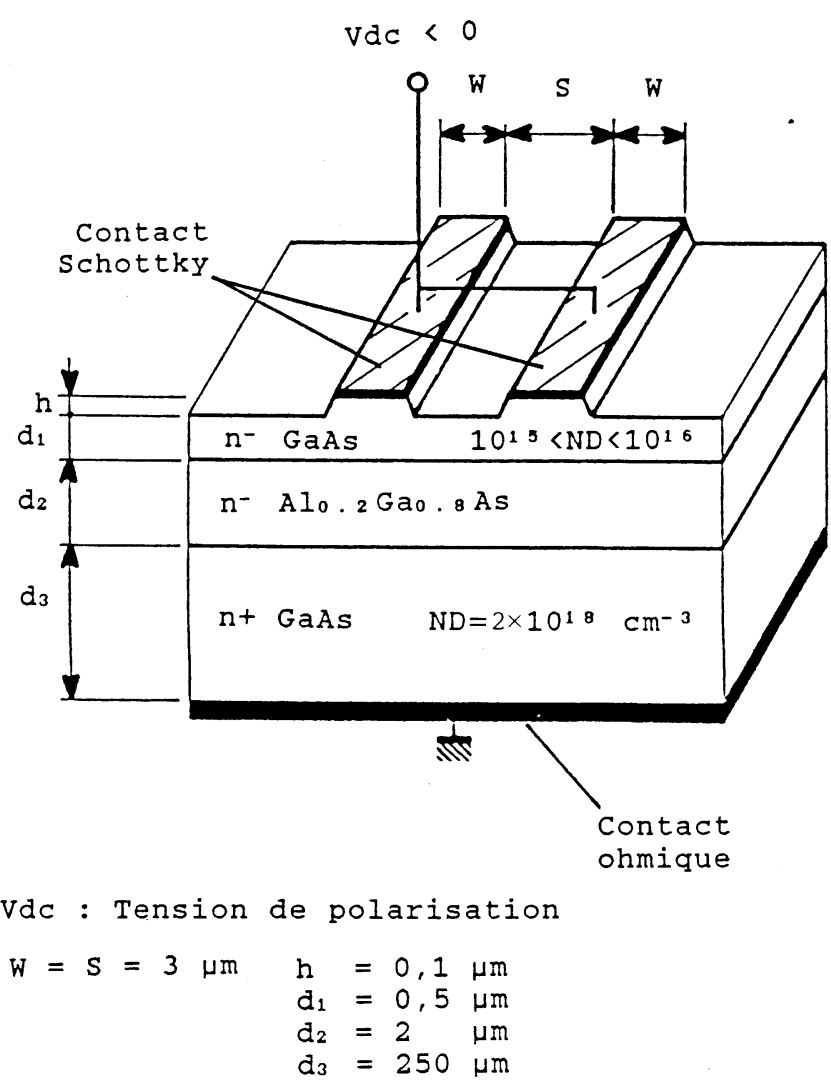

Fig. 1. - Région active du modulateur $\mathrm{MZ}$; l'indice microonde est accordé par $V_{\mathrm{dc}}$ à l'indice optique.

[Active section of $\mathrm{MZ}$ modulator ; the microwave effective index is tuned to the optical one by means of $V_{\mathrm{dc}}$.]

La composition de cette structure est classique en optique intégrée. Une couche guidante $\mathrm{n}^{-}$GaAs surmontant une couche d'indice optique plus faible $\mathrm{n}^{-} \mathrm{Al}_{x} \mathrm{Ga}_{1-x} \mathrm{As}$ constitue le guide optique planaire. Le confinement latéral de la lumière dans chaque bras de l'interféromètre est réalisé grâce aux « nervures » (rib) de $0,1 \mu \mathrm{m}$. Les couches $\mathrm{n}^{-} \mathrm{Al}_{x} \mathrm{Ga}_{1-x} \mathrm{As}$ et $\mathrm{n}^{-} \mathrm{GaAs}$ sont épitaxiées sur un substrat $\mathrm{n}^{+} \mathrm{GaAs}$.

Ce substrat est métallisé, le contact entre le métal et le semiconducteur dopé $\mathrm{n}^{+}$étant du type ohmique. En revanche, les électrodes déposées sur les 
guides « rib » constituent un contact Schottky avec le semiconducteur. Ces dernières forment, avec le plan de masse, une ligne de transmission microonde à deux rubans couplés.

La longueur active prévue, sur laquelle s'exerce l'interaction cumulative entre l'onde optique et le signal microonde est de l'ordre du centimètre.

Les signaux de commande du dispositif se décomposent en un signal microonde « utile », réalisant la modulation, et une tension de polarisation continue.

La tension de polarisation statique négative est appliquée entre chaque ruban métallique et le plan de masse de façon égale. Elle ne provoque donc aucune modulation de lumière globale en sortie de l'interféromètre. Le rôle de la polarisation est double : d'une part, elle crée une zone de déplétion sous les rubans, nécessaire pour un bon recouvrement du champ lumineux et du champ électrique microonde ; d'autre part, elle permet de régler la vitesse de phase microonde par l'intermédiaire de la largeur de déplétion.

A cette tension de polarisation, se superpose un « petit » signal microonde, transporté par la ligne à deux rubans couplés, et excité suivant un mode impair. Le champ électrique étant de sens opposé sous les rubans pour ce mode, il s'ensuit une modulation de l'intensité lumineuse en sortie par le signal microonde.

\section{Comportement de l'hétérostructure à barrière Schottky en microonde.}

\subsection{Propagation dans LeS STRUCTURES MIS ET À} BARRIÈRE SCHOTTKY. - Hasegawa et al. [5] ont étudié et expérimenté les caractéristiques de dispersion et de pertes de structures microruban sur un substrat formé d'une couche de semiconducteur ( $\mathrm{Si}$ ) et d'une couche isolante $\left(\mathrm{SiO}_{2}\right)$. Depuis lors, de nombreux chercheurs ont retrouvé ces résultats par d'autres méthodes de calculs [13, 14].

Les auteurs, dans la référence [5], ont d'abord considéré le cas d'un guide à deux plans parallèles, représenté à la figure 2 . Ils ont découvert que trois modes de propagation pouvaient exister suivant la fréquence de travail et la résistivité du semiconducteur. La condition d'existence de ces trois modes est explicitée par le diagramme de la figure 3.

La ligne de transmission MIS peut être modélisée, dans le cas général, par le schéma équivalent de la figure 4.1. Les condensateurs $\mathrm{C}_{1}$ et $\mathrm{C}_{2}$ représentent les couches d'isolant et de semiconducteur, séparés par des charges de polarisation interfaciale et situés entre les deux plans de métallisation. La conductance aux bornes de $\mathrm{C}_{2}$ tient compte de la conductivité du semiconduteur. L'inductance de ligne représente les courants longitudinaux dans les métallisations et dans le semiconducteur. Ces éléments varient forte-

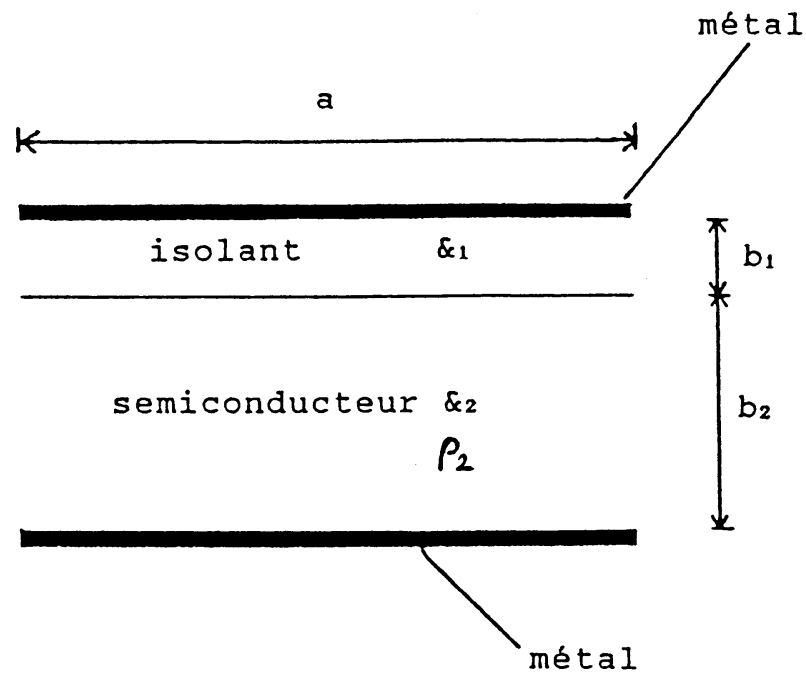

Fig. 2. - Structure MIS étudiée par Hasegawa et al. [5]. [MIS structure studied by Hasegawa et al. [5].]

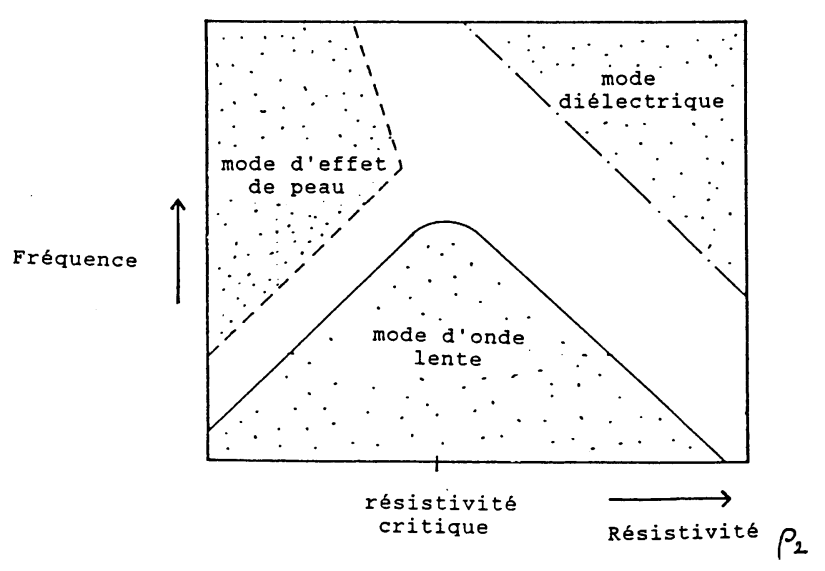

Fig. 3. - Les trois modes de propagation dans la structure figure 2 (d'après Hasegawa et al. [5]).

[The three modes of propagation within structure of figure 2 (from Hasegawa et al. [5]).]

ment en fonction de la fréquence; le mode de propagation n'est donc pas TEM ni quasi TEM.

Considérons en premier lieu le cas quasi statique ( $F$ tendant vers zéro) : toute la tension de ligne est développée aux bornes de $\mathrm{C}_{1}$; autrement dit, pratiquement toute l'énergie électrique se trouve dans la couche isolante. Quant à l'énergie magnétique, elle est située autour des métallisations et dans le semiconducteur, sièges des courants de lignes. Le schéma équivalent devient alors celui de la figure 4.2 , et la permittivité effective s'écrit :

$$
\varepsilon_{\mathrm{eff}}=n_{\mathrm{m} \text { eff }}^{2}=\varepsilon_{1} \cdot \varepsilon_{0} \cdot\left(b / b_{1}\right) .
$$

Dans les circuits intégrés, la couche isolante de passivation a une épaisseur $b_{1}$ beaucoup plus petite que l'épaisseur totale $b=b_{1}+b_{2}$. La vitesse de 


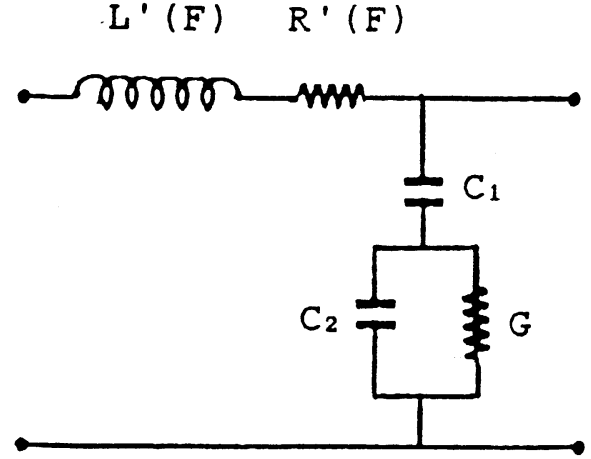

$$
\begin{aligned}
& C_{1}=\varepsilon_{0} \varepsilon_{1} \\
& C_{2}=\varepsilon_{0} \varepsilon_{2} \\
& G / b_{1} \\
& G=a /\left(b_{2}\right. \\
& \left.b_{2}\right)
\end{aligned}
$$

Fig. 4.1. - Cas général.

[General case.]

phase de l'onde $v_{\mathrm{p}}=\mathrm{C} / n_{\mathrm{m} \text { eff }}$ est alors très faible. Le mode correspondant à cette situation est appelé « mode d'onde lente ».

Envisageons maintenant le cas de plus hautes fréquences : deux situations distinctes vont apparâ̂tre suivant la résistivité du semiconducteur.

i) Pour les faibles valeurs de résistivité, l'effet de peau dans le semiconducteur devient prépondérant à mesure que la fréquence augmente. Cela se traduit par une relaxation de l'inductance de ligne (l'énergie magnétique n'est plus localisée dans toute la structure) et donc par une diminution de $n_{\mathrm{m} \text { eff }}$. La couche de semiconducteur peut être considérée, dans ce cas, comme un «mauvais» plan de masse. Le mode correspondant à cette situation est appelé «mode d'effet de peau » (voir Fig. 3).

ii) Pour les fortes valeurs de la résistivité, la couche semiconductrice se comporte sensiblement comme un diélectrique et l'énergie électrique n'est plus seulement localisée dans la couche isolante. On assiste à un phénomène de relaxation de la polarisation interfaciale à mesure que la fréquence augmente. Le résultat est là aussi la diminution de $n_{\mathrm{m} \text { eff }}$. Le mode correspondant s'appelle «mode diélectrique » (voir Fig. 3).

\subsection{APPLICATION AU MODULATEUR ÉLECTROOPTI-} QUE. - La structure réelle du modulateur comporte quelques différences par rapport au modèle planaire à deux plans métalliques parallèles étudié plus haut. - Les phénomènes évoqués restent qualitativement les mêmes, mais les résultats quantitatifs seront notablement modifiés. Il est néanmoins possible de prévoir dans quel sens ils évolueront.

3.2.1 Effet des rubans. - Lorsque l'on passe d'un guide à plans parallèles à une structure microruban,

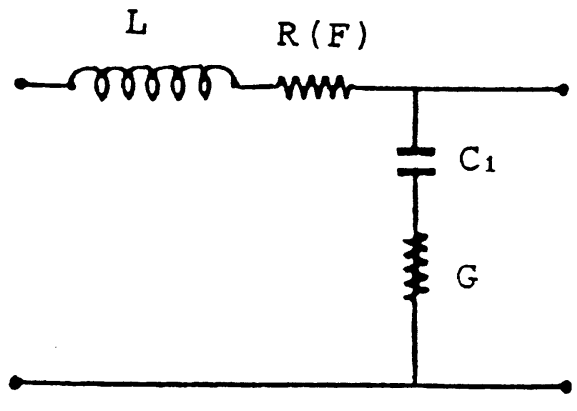

$L=\mu_{0} \mathrm{~b} / \mathrm{a}$

Fig. 4.2. - Cas quasi statique (mode d'onde lente).

[Quasi-static case (slow wave mode).]

les effets de bord sur le ruban doivent être pris en compte par des termes correctifs dans les expressions des constantes de propagation et de pertes [5]. Pour une ligne à deux rubans couplés, il faut en plus tenir compte du mode de propagation. En effet, il apparaît clairement sur la figure 5 que la ligne à rubans couplés aura un comportement plus proche du guide à plans parallèles pour le mode pair que pour le mode impair. Les lignes de champ, dans ce dernier cas, pénètrent moins dans le matériau semiconducteur et on peut s'attendre à des pertes plus faibles que pour le mode pair.

En outre, le rapport de l'épaisseur totale du substrat sur l'épaisseur de la couche isolante n'a plus de sens, avec le mode impair, pour définir l'indice microonde effectif lorsque le mode d'onde lente se propage. L'effet d'onde lente sera ici conditionné par le parcours des lignes de champ d'un ruban à l'autre. Etant donnée la faible distance séparant les rubans, l'effet d'onde lente sera moins marqué et la propagation sera donc moins dispersive pour le mode impair que pour le mode pair. Cet effet est favorable dans notre cas, où seul le mode impair induit une modulation de la lumière.

Par ailleurs, il convient de tenir compte de l'épaisseur des métallisations, qui peut être du même ordre de grandeur que la largeur des rubans, dans le cas où l'on veut réduire les pertes métalliques. On peut s'attendre alors à rencontrer davantage de lignes de champ dans l'air environnant, comme le montre la figure 5.3. Ceci contribue, pour le mode impair à diminuer encore l'effet d'onde lente [15].

3.2.2 Effet du contact Schottky. - Les zones de déplétion dans le modulateur (Fig. 6.1) jouent le rôle de la couche isolante de la structure figure 2 , à la différence près qu'elles n'occupent pas une région planaire. Les lignes de champ électrique restent 


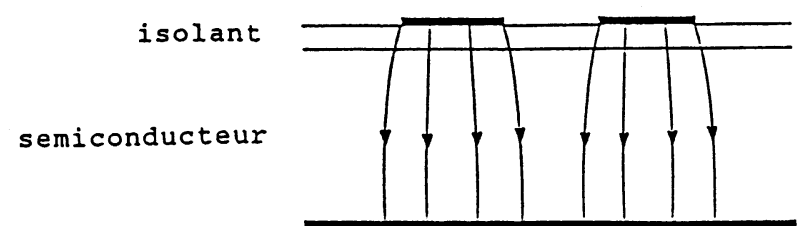

Fig. 5.1. - Structure du mod pair dans une ligne à rubans couplés.

[Even mode in a coupled-strip transmission line.]

isolant

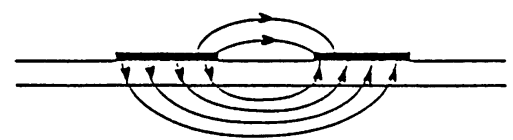

semiconducteur

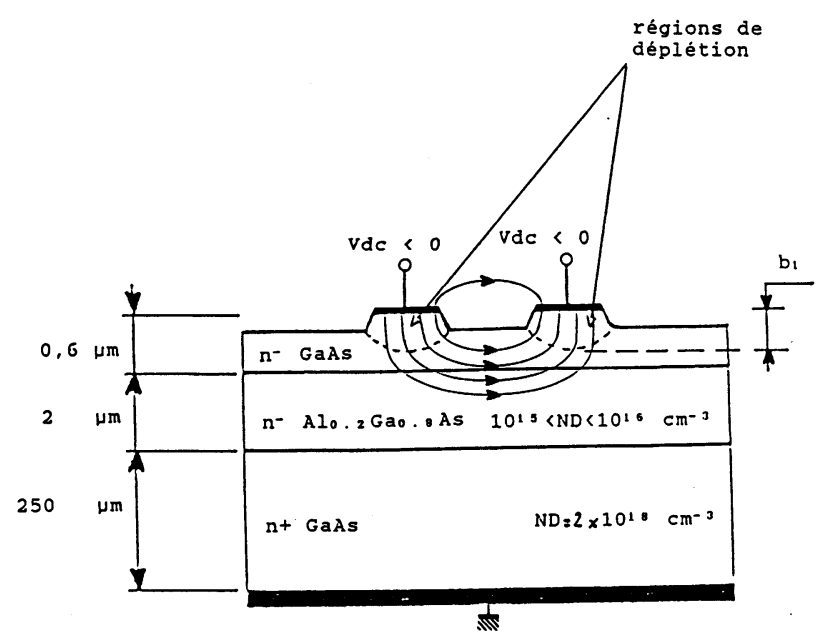

Fig. 6.1. - Allure des lignes de champ dans le modulateur.

[Feature of field lines within the modulator.]

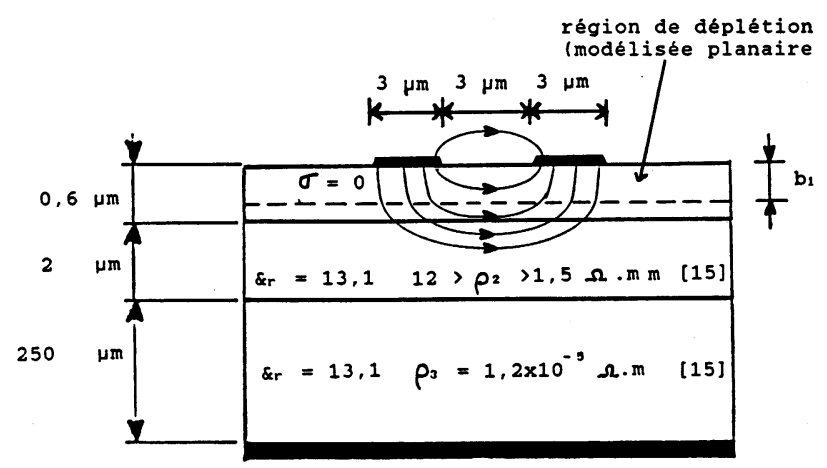

Fig. 6.2. - Modèle planaire de la structure figure 6.1.

[Planar layered model of structure figure 6.1.]

spectrale généralisée (MSG) ; les calculs correspondent bien aux résultats expérimentaux [12].

L'épaisseur de la région de déplétion est calculée d'après la relation [18] :

$$
b_{1}=\sqrt{\frac{2 \varepsilon_{0} \varepsilon_{\mathrm{r}}}{q N_{\mathrm{D}}}\left(V_{\mathrm{i}}-V_{\mathrm{dc}}-\frac{k T}{q}\right)}
$$

où $V_{\mathrm{i}}$ représente le potentiel interne et $V_{\mathrm{dc}}$ le potentiel appliqué.

3.2.3 Effet du substrat multicouche. - L'hétérostructure (Fig. 6.1) comporte trois couches de semiconducteurs de nature ou de dopage différent. Considérons tout d'abord l'hétérojonction isotype $\mathrm{n}^{-} \mathrm{Al}_{x} \mathrm{Ga}_{1-x} \mathrm{As} / \mathrm{n}^{-} \mathrm{GaAs}$. Avec $V_{\mathrm{dc}}<0$, cette hétérojonction est polarisée en direct. On peut donc lui supposer un comportement ohmique [19].

Pour les fortes polarisations, la zone de déplétion sous le contact Schottky atteint et dépasse l'hétérojonction, d'une façon similaire au phénomène observé en [20].

Pour les faibles polarisations $\left(V_{\mathrm{dc}}<0\right)$, l'accumulation d'électrons dans $\mathrm{n}^{-} \mathrm{GaAs}$ et la déplétion dans
En outre, le déphaseur commandé en tension de Neidert et al. [4] a également été remplacé par un modèle planaire pour être analysé par la méthode 
$\mathrm{n}^{-} \mathrm{Al}_{x} \mathrm{Ga}_{1-x} \mathrm{As}$ subsistent malgré la proximité $\mathrm{du}$ contact Schottky. L'étendue de ces zones (typiquement $100 \AA$ ) est très inférieure à la région de déplétion sous les rubans. On peut donc admettre que la propagation microonde est peu affectée par cet effet, cela revient à considérer les deux couches comme un seul et même matériau.

Finalement, le modèle étudié comporte une région de déplétion planaire et deux couches de semiconducteur dopées $\mathrm{n}^{-}$et $\mathrm{n}^{+}$. Il reste à considérer deux cas :

- La région de déplétion recouvre entièrement la couche $\mathrm{n}^{-}$.

Le modèle devient une structure MIS classique à faible résistivité et la caractéristique de dispersion révélera un passage du mode d'onde lente au mode d'effet de peau (Fig. 3). Si on excite le mode impair, le ralentissemnet de l'onde sera peu marqué; la propagation sera très peu dispersive, avec de faibles pertes et un indice effectif faible.

- La région de déplétion recouvre partiellement la couche $\mathrm{n}^{-}$.

La couche $\mathrm{n}^{+}$joue le rôle d'un plan de masse en comparaison à la couche $\mathrm{n}^{-}$. Le modèle de la figure 6.2 peut alors être limité aux régions où siège principalement l'énergie électromagnétique, à savoir la zone de déplétion et les régions $\mathrm{n}^{-}$. On a cette fois une structure MIS classique à forte résistivité, avec passage du mode d'onde lente au mode diélectrique sur la courbe de dispersion. L'effet d'onde lente sera plus important que dans le cas précédent, d'où un indice effectif plus élevé et augmentant lorsque $V_{\mathrm{dc}} \rightarrow 0$. Parallèlement à ceci, la propagation sera plus dispersive et les pertes plus élevées.

\section{Optimisation et résultats.}

La première étape consiste à dimensionner les rubans et leur écartement de telle façon que l'indice microonde soit inférieur à l'indice optique lorsque la région de déplétion occupe toute la région $\mathrm{n}^{-}$. Ceci exige que $W$ et $S$ (voir Fig. 1) soient très faibles, afin de diminuer la capacité de ligne [8]. Nous constatons d'après la figure 7 (courbe $b_{1}=2,6 \mu \mathrm{m}$ ) que ce résultat est atteint pour $W=S=3 \mu \mathrm{m}$.

La suite de l'étude appartient au domaine de l'optoélectronique et définit la composition de l'hétérostructure avec cette nouvelle contrainte.

4.1 CONCEPTION DU MODUlATEUR EN OPTIQUE INTÉGRÉE. - L'indice optique dans GaAs vaut 3,59 à la longueur d'onde $\lambda=0,9 \mu \mathrm{m}$, tandis qu'il s'écrit dans $\mathrm{Al}_{x} \mathrm{Ga}_{1_{-x}} \mathrm{As}$ :

$$
n(x)=3,59-0,710 x+0,091 x^{2}
$$

Soit :

$$
n(0,2)=3,4516 \text {. }
$$

IIIDICE EFFECTIF MICROONDE $n_{\text {meff }}$

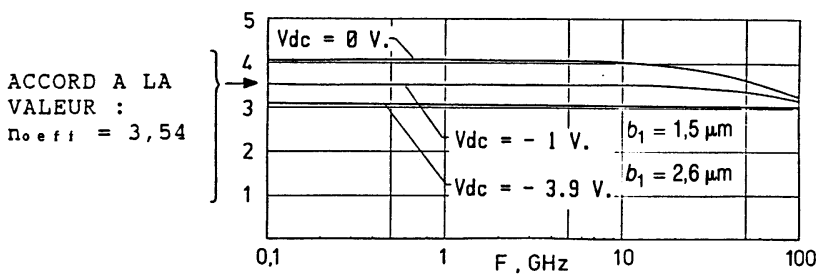

Fig. 7.1. - Caractéristique de dispersion de la structure figure 6.2 pour $N D=10^{15} \mathrm{~cm}^{-3}$.

[Dispersion characteristics of structure figure 6.2 for $N D=10^{15} \mathrm{~cm}^{-3}$.]

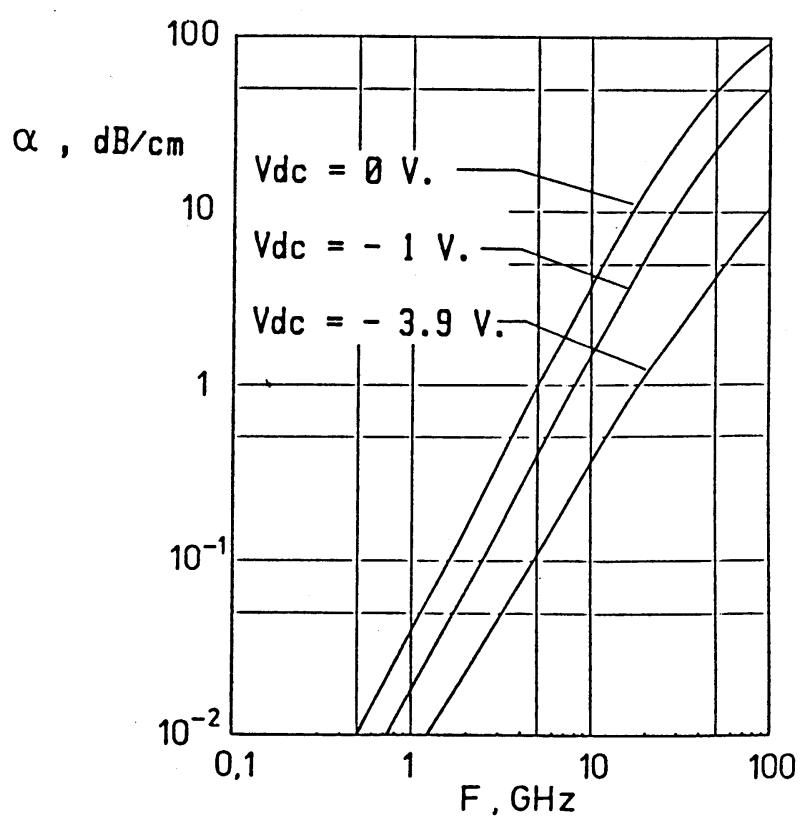

Fig. 7.2. - Caractéristique de pertes de la structure figure 6.2 pour $N D=10^{15} \mathrm{~cm}^{-3}$.

[Losses characteristics of structure figure 6.2 for $N D=$ $10^{15} \mathrm{~cm}^{-3}$.]

On vérifie [10] que le guide « rib » est monomode dans ces conditions avec $d_{1}+h=0,6 \mu \mathrm{m}$ (voir Fig. 1).

Pour obtenir un bon confinement vertical de la lumière, on souhaite que l'épaisseur $\left(d_{2}=2 \mu \mathrm{m}\right)$ de la couche $\mathrm{Al}_{0,2} \mathrm{Ga}_{0,8} \mathrm{As}$ représente 10 fois la distance pour laquelle les champs décroissent dans cette couche d'un facteur $1 / e$.

Cette condition est réalisée avec un guide planaire d'épaisseur $d_{1}=0,5 \mu \mathrm{m}$, conduisant à un indice effectif de 3,53194 et une diminution 1/e des champs à une profondeur de $0,19 \mu \mathrm{m}$ dans le milieu $\mathrm{Al}_{0,2} \mathrm{Ga}_{0,8}$ As.

Le confinement horizontal peut alors être évalué. Une nervure de hauteur $h=0,1 \mu \mathrm{m}$ reste très efficace, puisque l'indice calculé pour un guide planaire d'épaisseur $d_{1}+h=0,6 \mu \mathrm{m}$, et recouvert 
d'or, vaut 3,54289 , ce qui représente un écart relativement important par rapport au guide d'épaisseur $d_{1}=0,5 \mu \mathrm{m}$.

La méthode de l'indice effectif [10] nous confirme cette propriété : pour un guide « rib » large de $3 \mu \mathrm{m}$, le champ subit une décroissance $1 / e$ à une distance $0,6 \mu \mathrm{m}$ de chaque bord du guide. Le couplage par onde évanescente entre les deux guides sera donc négligeable si ceux-ci sont séparés d'une distance $S=3 \mu \mathrm{m}$. Plus précisément, la longueur de couplage évaluée par la théorie des modes couplés est $L$ \# $18 \mathrm{~cm}$.

L'indice effectif optique finalement obtenu vaut pour le mode fondamental TEO : $n_{\mathrm{o} \text { eff }}=3,54$. L'atténuation due aux électrodes métalliques est plus faible pour ce mode que pour un mode TM. Elle peut toutefois être encore réduite, sans modifier fondamentalement le principe de l'accord électronique de l'indice microonde, en ajoutant une couche tampon (\# $1000 \AA$ ) en $\mathrm{SiO}_{2}$ ou $\mathrm{Si}_{3} \mathrm{~N}_{4}$.

4.2 CalCuls ET RÉSultats EN MiCROONDE. Les calculs en microonde sont menés à l'aide de la méthode spectrale généralisée (MSG) [12], qui permet une modélisation très complète des technologies planaires telles que celle de la figure 6. La MSG est une méthode rigoureuse à dominante analytique. Moyennant un développement analytique important, elle conduit à des temps de calcul sur ordinateur tout à fait modérés.

Le principe de la MSG consiste à ramener toutes les équations du problème à une seule et unique équation - dite «équation fondamentale » - dans le plan de métallisation (domaine de Fourier), qui s'écrit :

$$
I_{\mathrm{T}}(\alpha)=G_{\mathrm{T}}(\alpha) \cdot E_{\mathrm{T}}(\alpha)
$$

où :

$I_{\mathrm{T}}(\alpha)$ représente la densité de courant dans le plan de métallisation.

$E_{\mathrm{T}}(\alpha)$ est le champ électrique tangentiel dans ce même plan.

$G_{\mathrm{T}}(\alpha)$ est une matrice $2 \times 2$ qui s'exprime analytiquement et est connue sous le nom de matrice de Green de la MSG.

L'équation fondamentale est généralement résolue numériquement sur ordinateur par une méthode de moment classique [21]. Sa résolution donne les caractéristiques de dispersion et de pertes de tous les modes susceptibles de se propager, ainsi que la carte des champs dans le plan de métallisation. Partant de ces valeurs, on peut calculer la carte des champs dans toute la structure.

Nous avons appliqué la MSG au modèle de la figure 6.2 pour plusieurs dopages $N D$ de la couche guidante. Les caractéristiques de dispersion et de pertes pour $N D=10^{15} \mathrm{~cm}^{-3}$ et $N D=10^{16} \mathrm{~cm}^{-3}$ sont représentées aux figures 7 et 8 .

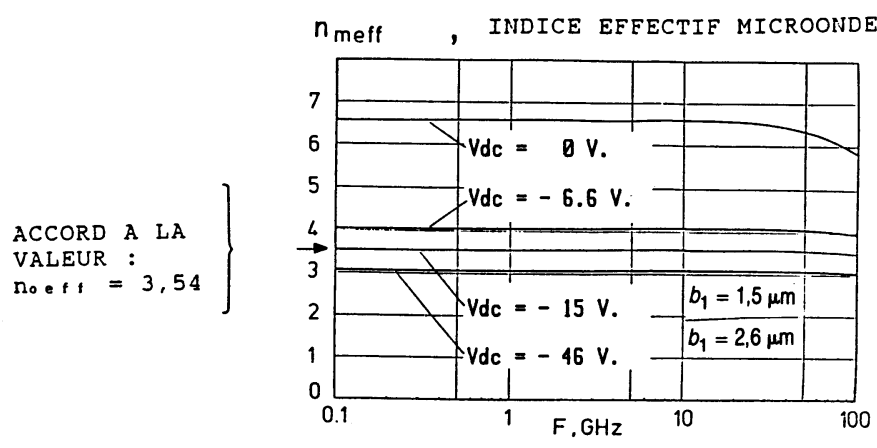

Fig. 8.1. - Caractéristique de dispersion de la structure figure 6.2 pour $N D=10^{16} \mathrm{~cm}^{-3}$.

[Dispersion characteristics of structure figure 6.2 for $N D=10^{16} \mathrm{~cm}^{-3}$.]

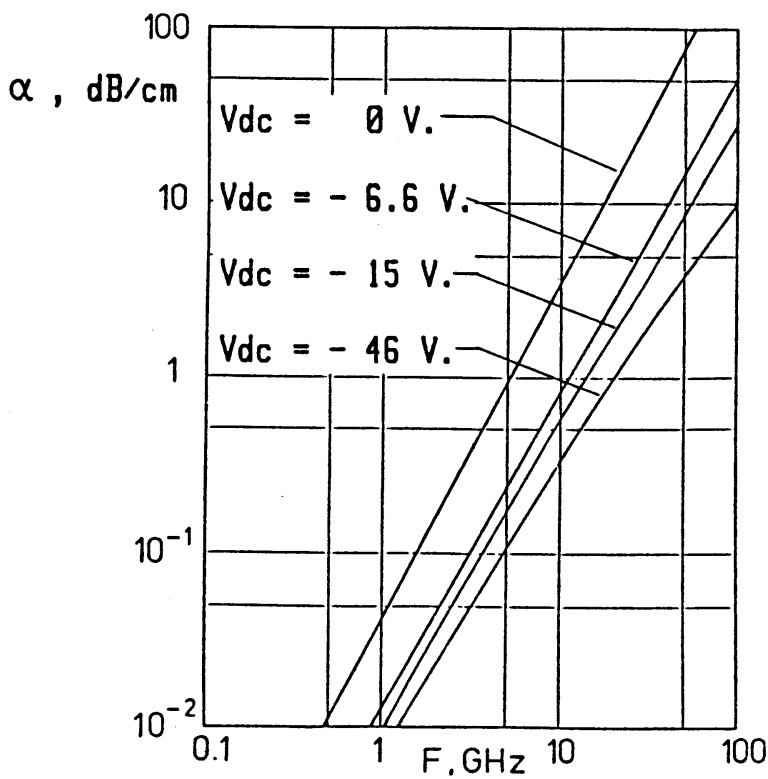

Fig. 8.2. - Caractéristique de pertes de la structure figure 6.2 pour $N D=10^{16} \mathrm{~cm}^{-3}$.

[Losses characteristics of structure figure 6.2 for $N D=$ $10^{16} \mathrm{~cm}^{-3}$.]

Les résultats correspondent aux prévisions théoriques exposées plus haut. L'indice effectif microonde est très peu dispersif pour $b_{1}=2,6 \mu \mathrm{m}$ (déplétion totale de la couche active). Il augmente lorsque $V_{\mathrm{dc}} \rightarrow 0$, si bien qu'il atteint la valeur de l'indice effectif optique $\left(n_{\mathrm{o} \text { eff }}=3,54\right)$ pour $V_{\mathrm{dc}}=-1 \mathrm{~V}$ et $V_{\mathrm{dc}}=-15 \mathrm{~V}$ avec $N D=10^{15} \mathrm{~cm}^{-3}$ et $N D=10^{16}$ $\mathrm{cm}^{-3}$ respectivement. L'accord des indices effectifs correspond dans les deux cas à une déplétion $b_{1} \# 1,5 \mu \mathrm{m}$ [18]. A ce stade, la courbe est légèrement plus dispersive, car l'effet de ralentissement de l'onde commence à se faire sentir en basse fréquence. On dénote d'ailleurs une plus grande dispersion pour le dopage le plus faible, car les lignes de champ pénètrent davantage dans le milieu $\mathrm{n}^{-}$(voir 
Fig. 6.1). L'indice effectif reste malgré tout constant et pratiquement égal à $n_{\text {off pour } 0<F<20 \mathrm{GHz}}$ avec $N D=10^{15} \mathrm{~cm}^{-3}$ et pour $0<F<50 \mathrm{GHz}$ avec $N D=10^{16} \mathrm{~cm}^{-3}$.

En revanche, la courbe correspondant à $V_{\mathrm{dc}}=0 \mathrm{~V}$ donne un effet d'onde lente beaucoup plus marqué (voir Eq. (1)) pour le dopage le plus élevé car la valeur de $b_{1}$ est plus faible dans ce cas.

L'écart maximal $\delta n=\left|n_{\mathrm{m} \text { eff }}-n_{\mathrm{o} \text { eff }}\right|$ relevé sur les courbes $b_{1}=1,5 \mu \mathrm{m}$ (Fig. 7.1 et 8.1) vaut dans la bande $0-100 \mathrm{GHz}$ :

$$
\begin{array}{ll}
\delta n_{\max }=0,17 & \left(N D=10^{15} \mathrm{~cm}^{-3}\right) \\
\delta n_{\max }=0,045 & \left(N D=10^{16} \mathrm{~cm}^{-3}\right) .
\end{array}
$$

Pour évaluer la limitation de la largeur de bande de modulation due à la seule désadaptation des indices, considérons le cas plus simple d'un modulateur long de $1 \mathrm{~cm}$ et tel que $\delta n$ soit constant et égal à $\delta n_{\max }$. On trouve pour les deux valeurs de $N D$ une largeur de bande supérieure à $100 \mathrm{GHz}$ [22], ce qui dépasse notre domaine d'investigation.

D'autres sources de limitation de la largeur de bande interviennent, comme les pertes microonde en ligne et la désadaptation des impédances caractéristiques.

Les pertes se décomposent en pertes métalliques, variant comme $\sqrt{f}$, et en pertes dans les couches de semiconducteur, représentées aux figures 7.2 et 8.2. Ces dernières sont relativement modérées pour la tension de polarisation réalisant $n_{\mathrm{m} \text { eff }}=n_{\mathrm{o} \text { eff }}$. Elles sont cependant plus élevées pour le dopage le plus faible, car l'onde pénètre davantage dans le milieu $\mathrm{n}^{-}$GaAs dissipatif $\left(4 \mathrm{~dB} / \mathrm{cm}\right.$ pour $N D=10^{15}$ $\mathrm{cm}^{-3}$ et $2 \mathrm{~dB} / \mathrm{cm}$ pour $N D=10^{16} \mathrm{~cm}^{-3}$, à $20 \mathrm{GHz}$ ).

Il est clair que les pertes deviennent une cause importante de la limitation de la largeur de bande de modulation, lorsque le synchronisme des ondes optique et hyperfréquence est réalisé. Les pertes dans les couches semiconductrices peuvent être diminuées en remplaçant le substrat $\mathrm{n}^{+}$par un substrat semi-isolant sur lequel serait déposée une couche de quelques microns de semiconducteur dopé $\mathbf{n}^{+}$. Ceci apporte néanmoins des problèmes technologiques supplémentaires, inhérents à la prise de contact sur la couche $\mathrm{n}^{+}$pour appliquer la polarisation statique.

Quant aux pertes métalliques, elles doivent être réduites par un épaississement des métallisations. Cette nécessité est d'autant plus importante que la largeur des rubans doit être la plus petite possible pour assurer le synchronisme des ondes optique et hyperfréquence.

Ceci amène l'optimisation du modulateur vers une autre forme de compromis : en effet, l'augmentation de l'épaisseur des rubans réduit l'effet d'onde lente (voir paragraphe 3.2), tandis que l'augmentation de leur largeur accroît cet effet. On peut alors espérer retrouver le synchronisme des ondes pour des rubans plus larges et plus épais. En contrepartie, la puissance de commande nécessaire sera accrue, en raison d'une part plus importante d'énergie électromagnétique dans l'air environnant (voir Fig.5.3). Cette partie de l'étude fait l'objet d'une seconde étape dans la conception du modulateur.

L'optimisation du dopage de la couche guidante est ensuite abordée. La figure 9.1 représente la

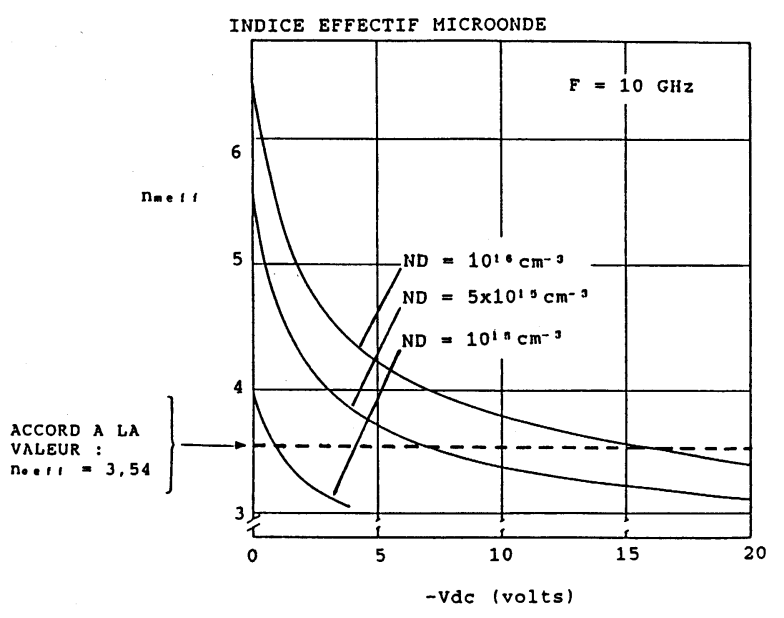

Fig. 9.1. - Variation de l'indice effectif microonde en fonction de la polarisation statique, pour plusieurs dopages de la couche active.

[Effective microwave index versus static bias, for several doping levels of the active layer.]

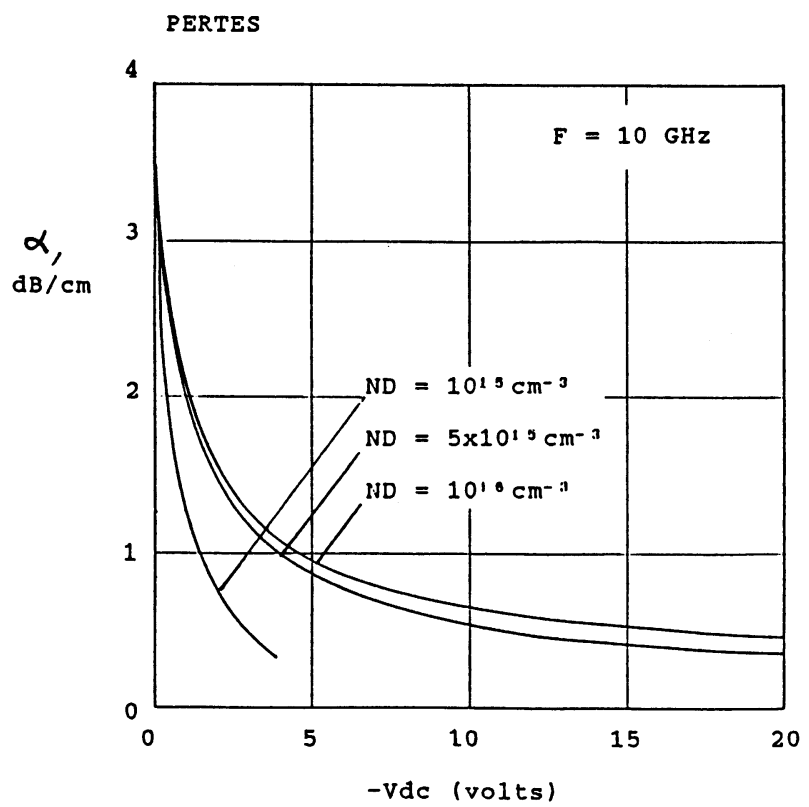

Fig. 9.2. - Evolution des pertes en fonction de la polarisation statique, pour plusieurs dopages de la couche active.

[Evolution of losses versus static bias, for several doping levels of the active layer.] 
variation de $n_{\mathrm{m} \text { eff }}$ en fonction de $V_{\mathrm{dc}}$ à la fréquence de $10 \mathrm{GHz}$, pour plusieurs valeurs de $N D$. Il apparaît que le réglage $n_{\mathrm{m} \text { eff }}=n_{\mathrm{o} \text { eff }}$ est moins sensible - et moins délicat - pour $N D=10^{16} \mathrm{~cm}^{-3}$ que pour $N D=10^{15} \mathrm{~cm}^{-3}$. Un dopage à $5 \times 10^{15} \mathrm{~cm}^{-3}$ semble être un compromis acceptable de ce point de vue. La tension d'accord serait alors de $-7 \mathrm{~V}$.

La figure 9.2 représente les pertes dans les matériaux semiconducteurs en fonction de la polarisation. Les pertes augmentent fortement lorsque $V_{\mathrm{dc}} \rightarrow 0$, car $b_{1}$ diminue et le champ électromagnétique s'étend davantage dans le semiconducteur. Si on s'intéresse pour chaque courbe au point de polarisation réalisant l'accord des indices, les pertes sont plus faibles pour le dopage le plus élevé $(1,3 \mathrm{~dB}$ au point $V_{\mathrm{dc}}=-1 \mathrm{~V}$ pour $N D=10^{15} \mathrm{~cm}^{-3}$ et $0,7 \mathrm{~dB}$ à $V_{\mathrm{dc}}=-7 \mathrm{~V}$ pour $\left.N D=5 \times 10^{15} \mathrm{~cm}^{-3}\right)$.

4.3 DOMAINE DE VALIDITÉ. - L'analyse microonde présentée ci-dessus a été faite dans l'hypothèse des petits signaux, permettant de négliger la variation de la largeur de déplétion en fonction de la valeur instantanée du signal de modulation. Le cas des grands signaux a néanmoins une réalité concrète, surtout lorsque des puissances de commande importantes sont nécessaires.

Il convient de discuter en premier lieu le cas simple d'un modulateur de phase basé sur le même principe, à savoir formé d'une électrode monoruban sous lequel est guidée la lumière, une polarisation statique étant appliquée entre ruban et plan de masse, donc parfaitement superposée au mode fondamental microonde propagé.

Dans le cas d'une polarisation inverse du contact Schottky, on peut alors s'attendre à des variations notables de la largeur de déplétion jusqu'à des fréquences très élevées puisque la durée de vie des porteurs ne constitue pas ici de limitation (à l'inverse du cas d'une polarisation directe [23]).

Du point de vue optique, cela fait intervenir d'autres effets, créant également une modulation de la lumière. Parmi ceux-ci, citons l'effet plasma induisant une variation de l'indice optique en fonction de la concentration des charges libres; l'indice effectif optique de la structure dépend donc de la largeur de déplétion [24].

Du point de vue microonde, le problème devient non linéaire puisque la vitesse de phase de l'onde dépend du niveau instantané du signal [25].

Revenons maintenant au cas de notre structure à deux rubans couplés et plan de masse (Fig. 6.1). L'étude serait plus compliquée, car le signal microonde s'applique entre les deux rubans, tandis que la polarisation statique s'applique entre chaque ruban et le plan de masse. Si une variation de la largeur de déplétion peut être induite par le signal hyperfréquence, celle-ci serait certainement antisymétrique, d'où une modulation de la lumière due aux effets décrits ci-dessus plus importante.

\section{Conclusion.}

La faisabilité d'un modulateur électrooptique, dans lequel l'indice effectif microonde peut être commandé par une tension de polarisation continue, est déduite d'une analyse microonde rigoureuse des caractéristiques de dispersion et de pertes de ce nouveau composant. Ce dernier est la synthèse d'un déphaseur microonde commandé en tension et d'un modulateur électrooptique du type interféromètre de Mach-Zehnder sur GaAs/AlGaAs.

Les calculs ont été effectués pour réaliser l'optimisation des dimensions de la structure d'électrode, de l'épaisseur des différentes couches et enfin du dopage de ces dernières.

Il s'avère que la structure d'électrode (largeur des rubans additionnée à leur espacement) doit être la plus petite possible par rapport à l'épaisseur totale $\mathrm{du}$ dispositif afin de diminuer l'indice effectif microonde. Le découplage optique entre les deux branches de l'interféromètre doit alors être assuré grâce à la présence d'une couche de confinement $\mathrm{Al}_{x} \mathrm{Ga}_{1-x} \mathrm{As}$.

Lorsque la couche guidante est totalement dépeuplée, l'indice effectif microonde $n_{\mathrm{m}}$ eff est inférieur à l'indice effectif optique $n_{\mathrm{o} \text { eff }}$. La diminution de la polarisation $\left(V_{\mathrm{dc}} \rightarrow 0\right)$ entraîne alors une augmentation de $n_{\mathrm{m} \text { eff }}$, si bien que la condition d'accord $n_{\mathrm{m} \text { eff }}=n_{\mathrm{o} \text { eff }}$ peut être atteinte pour plusieurs dopages $N D$ de la couche guidante. Dans chaque cas, la propagation microonde est très peu dispersive jusqu'à $100 \mathrm{GHz}$, avec $n_{\mathrm{m} \text { eff }}$ égalant pratiquement $n_{\text {o eff }}$.

Le dopage de la couche $\mathrm{n}^{-}$joue un double rôle : sur la valeur de la tension $V_{\mathrm{dc}}$ d'accord, ainsi que sur la profondeur de pénétration de l'onde hyperfréquence. Un très faible dopage rend le réglage de $V_{\mathrm{dc}}$ plus sensible et une propagation moins dispersive que pour un dopage plus élevé, mais les pertes sont plus élevées. Ces dernières restent malgré tout modérées, inférieures à $2 \mathrm{~dB} / \mathrm{cm}\left(N D=10^{16} \mathrm{~cm}^{-3}\right)$ et à $4 \mathrm{~dB} / \mathrm{cm}\left(N D=10^{15} \mathrm{~cm}^{-3}\right)$ jusqu'à $20 \mathrm{GHz}$.

Le problème des pertes devient prépondérant pour l'efficacité du modulateur et de sa bande passante. Une détermination plus affinée de certains paramètres géométriques peut être envisagée par la suite : Utilisation d'un substrat semi-isolant pour réduire les pertes dans le semiconducteur et épaississement des rubans pour diminuer les pertes métalliques. Ceci doit faire l'objet d'une étude ultérieure. 


\section{Bibliographie}

[1] Charil, J., Lesterlin, D., Correc, P., Rose, B., Gilleron, M., Chaminant, G., Bouley, J. C., « Lasers $1,5 \mu \mathrm{m}$ à réaction distribuée et à ruban enterré obtenus par épitaxie hybride EPL/OM-CVD». Journées Nationales Microélectronique III-V, Aussois, France, 7-9 janvier 1987.

[2] Gee, C. M., Thurmond, G. D., Yen, H. W., « $17 \mathrm{GHz}$ Bandwidth Electro-optic Modulator » Appl. Phys. Lett. 43 (1983) 998-1000.

[3] Buchmann, P., Kaufmann, H., Melchior, H., Guekos, G., «Broadband Y-branch Electrooptic GaAs Waveguide Interferometer for 1,3 $\mu \mathrm{m}$ » Appl. Phys. Lett. 46 (1985) 462-464.

[4] Neidert, R. E., Krowne, C. M., « Voltage Variable Microwave Phase Shifter " Electron. Lett. 21 (1985) 636-638.

[5] Hasegawa, H., Furukawa, M., Yanai, H., « Properties of Microstrip Line on $\mathrm{Si}_{-} \mathrm{SiO}_{2}$ system » IEEE Trans. MTT 19 (1971) 869-881.

[6] JAFFE, J. M., «A High Frequency Variable Delay Line ». IEEE Trans. ED 19 (1972) 1292-1294.

[7] Walker, R. G., Carter, A. C., « GaAs/GaAlAs Heterostructure Electro-Optic Modulators and Switches ». IEEE Workshop on Int. Opt. \& Related Tech., Florence (1984).

[8] Fukuoka,Y., Shiн, Y. C., IтOH, T., « Analysis of Slow-Wave Coplanar Waveguide for Monolithic Integrated Circuits » IEEE Trans. MTT 31 (1983) 567-573.

[9] Tedjini, S., Rauly, D., Pic, E., « Optimisation de la Largeur de Bande d'un Modulateur Electrooptique " $7^{\mathrm{e}}$ Journées Nationales d'Optique Guidée, Nice, 3-4 avril 1986.

[10] TAMIR, T., Integrated Optics (Springer-Verlag, Berlin, N. Y.) 1975.

[11] Izutsu, M., Yamane, Y., Sueta, T., « Broad-Band Traveling-Wave Modulatoir Using a $\mathrm{LiNbO}_{3}$ Optical Waveguide » IEEE $Q E 13$ (1977) 287295.

[12] Tedjini, S., Rauly, D., Saguet, P., « Generalized Spectral Domain Technique, Theory and Appli- cations » Proc. 16th E.M.C., Dublin, 8-12 sept. 1986.

[13] Kennis, P., Faucon, L., « Rigorous Analysis of Planar Transmission Lines "Electron. Lett. 17 (1981) 454-456. Journées Nationales Microélectroniques III-V.

[14] Aubourg, M., Villotte, J. P., Godon, F., Garault, Y., "Finite Element Analysis of Lossy Waveguides. Application to Microstrip Lines on Semiconductor Substrate » IEEE Trans. MTT 31 (1983) 326-331.

[15] Seguinot, C., Pribetich, P., Kennis, P., « Propriétés des lignes microniques déposées sur AsGa » Journées Nationales Microélectronique III-V, Aussois, France 7-9 janvier 1987.

[16] SнIH, Y. C., Iтон, T., « Analysis of printed transmission lines for monolithic integrated circuits » Electron. Lett. 18 (1982) 585-586.

[17] Tzuang, C. K., Iтон, T., « Finite-Element analysis of slow-wave Schottky contact printed lines» IEEE Trans. MTT 34 (1986) 1483-1489.

[18] SzE, S. M., Physics of Semiconductor Devices (2nd Edition) (J. Wiley \& Sons, Inc) 1981.

[19] Chandra, A., Eastman, L. F., « Rectification at $\mathrm{n}^{-}$-n GaAs : (Ga, Al)As Heterojunctions, Electron. Lett. 15 (1979) 90-91.

[20] LANG, D. V., LogAN, R. A., «A Search for Interface States in an LPE GaAs $/ \mathrm{Al}_{x} \mathrm{Ga}_{1-x}$ As Heterojunction, Appl. Phys. Lett. 31 (1977) 683-684.

[21] Harrington, R. F., Field Computation by Moment Methods (McMillan Co, New York) 1968.

[22] AlfERnEss, R. C., « Waveguide Electrooptic Modulators » IEEE trans. MTT 30 (1982) 1121-1137.

[23] Mikami, O., NaKagome, H., « Waveguided optical switch in InGaAs /InP using free-carrier plasma dispersion " Electron. Lett. 20 (1984) 228-229.

[24] Alping, A., Wu, X. S., Hausken, T. R., Coldren, L. A., « Highly efficient waveguide phase modulator for integrated optoelectronics » Appl. Phys. Lett. 48 (1986) 1243-1245.

[25] Fallside, F., Bickley, D. T., "Shock waves in a nonlinear delay line $»$ Electron. Lett. 2 (1966) 57. 